\title{
REVIEW
}

\section{Patient monitoring alarms in the ICU and in the operating room}

\author{
Felix Schmid*, Matthias S Goepfert, Daniel A Reuter \\ This article is one of ten reviews selected from the Annual Update in Intensive Care and Emergency Medicine 2013 and co-published as a series \\ in Critical Care. Other articles in the series can be found online at http://ccforum.com/series/annualupdate2013. Further information about the \\ Annual Update in Intensive Care and Emergency Medicine is available from http://www.springer.com/series/8901.
}

\section{Introduction}

Historically, the word 'alarm' originates from the Latin, 'ad arma', or the French, 'à l'arme', which can be translated into 'to your weapons.' Hence, the word indicates a call for immediate action, for attack or for defense. Alarms have existed ever since humans have lived in groups. Some of the first documented alarms are watchmen on towers in the Middle Ages, who warned of fires or enemies by ringing bells. Warning fires provided a visual alert to enemy attacks, visible across long ranges and enabling an early reaction of armed forces. Today, comparable systems are available that send warning-SMSs (Short Message Service) of nearing tsunamis to mobile phones [1].

In complex fields of work like aviation, mining, anesthesiology, and intensive care medicine - and here particularly with regard to monitoring of vital functions alarms are ubiquitous and have been the subject of medical, technical, and psychological research for decades [2,3]. Monitoring of vital functions and function of life-support devices is essential for critically ill patients, although real evidence based data are missing. However, modern patient monitors and implemented risk management (including alarms) must be constructed in accordance to approved and current international standards IEC 60601-1-11 and IEC 80001-1 [4,5]. Tinker et al. surveyed 1,175 anesthetic-related closed malpractice claims from 17 professional liability insurance companies. It was determined that $31.5 \%$ of the negative outcomes could have been prevented by use of additional monitors. The authors concluded that monitoring with adequate thresholds appeared able to improve patient outcomes [6]. Cooper et al. showed in the 1980s that $70 \%$ of all anesthesia-related critical incidents were caused by

*Correspondence: feschmid@uke.de

Department of Anesthesiology, Center of Anesthesiology and Intensive Care Medicine, Hamburg Eppendorf University Hospital, Martinistr. 52, 20246 Hamburg, Germany human error [7]. Similar data are available from the aviation industry [8]. Inevitable mistakes may be corrected in time if detected by a monitoring system (including alarms) before physiological variables run out of range.

An alarm is an automatic warning that results from a measurement, or any other acquisition of descriptors of a state, and indicates a relevant deviation from a normal state [9]. Loeb surveyed the reaction of anesthesiologists to relevant changes in monitoring parameters, and showed that anesthesiologists needed a mean time of 61 seconds to recognize a change in the parameters; $16 \%$ of the changes were unrecognized for over 5 minutes [10]. In contrast, Morris and Montano studied the reaction of anesthesiologists to optical and acoustic warnings during maintenance of general anesthesia. The anesthesiologists showed a reaction time of 6 seconds to optical warnings and 1 second to acoustic warnings [11]. An ideal alarm should only detect immediate or threatening danger that requires prompt attention. The alarm design should adequately represent the underlying situation. The announcement of the alarm should be instantly perceptible in critical situations. Additionally, the user should be informed of circumstances that impair the reliability of the alarming system.

In addition to these general properties, device alarms have various goals, which follow a certain hierarchy [12]:

- Detection of life-threatening situations: The detection of life-threatening situations was the original purpose of monitor alarms. False negative alarms are not acceptable in such situations because of the danger of severe patient harm or death.

- Detection of imminent danger: The early detection of gradual change that might indicate imminent danger.

- Diagnostic alarms: These alarms indicate a pathophysiological condition (e. g., shock) rather than warning of 'out-of-range' variables.

- Detection of life-threatening device malfunction: This ability is essential for all life-support devices, which 
must recognize malfunctions, such as disconnection from the patient, occlusion of the connection to the patient, disconnection from power, gas, or water supply, and internal malfunction.

- Detection of imminent device malfunction: The early detection of device-related problems that could result in malfunction is an integral part of many therapeutic devices. These warning mechanisms range from simple aspects (e. g., low-battery warnings) to complex algorithms and sensors that track the wear of respiratory valves.

\section{Alarm design}

Alarms are typically displayed in two ways or as a combination of both:

\section{Acoustic}

The alarm is given as a warning sound. Most manufacturers distinguish the priority of an alarm with different signals. Intuitive alarms with different tone sequences (e. g. 'short-long-short' for 'ven-ti-late') have been the object of research but have not found their way into routine clinical practice. Alarms directly mentioning organ systems, device hardware, or parts of it (e. g., ventilation or circulation) or alarms with direct labeling of the physiological problem ('bloodpressure' or 'oxygen') have also not been introduced into practice [13].

2. Visual

Visual alarms involve mostly flashing or coloring of the related parameter in an eye-catching manner. Some systems provide integrated displays of several parameters. One example is a spider-display, which shows the relationship of different parameters in a stylized spider web. Such applications can be useful to display different parameters in context. Compared to other professions in industry and aviation, adoption of such new displays in healthcare has been slow.

\section{Alarm-related problems}

Alarms help to prevent patient harm by providing rapid recognition of and reaction to critical situations, but only if they are not 'false alarms'. Medical progress leads to an increasing number of 'monitorable' parameters and thus an increasing number of possible alarms.

\section{False alarms}

In medicine, false alarms are conventionally defined as alarms without clinical or therapeutic consequence. Today's monitoring systems are still designed using a 'better-safe-than-sorry'-logic: A large number of false alarms are accepted rather than risking missing one valid alarm [14]. Alarms can be differentiated into technically correct/technically false and clinically relevant/clinically not relevant. Alarms can be classified as technically correct, if they are based upon a technically correct measurement. Technically false alarms are not based on a technically correct measurement (e. g., interference with pulse oximetry caused by ambient light). Because not all technically correct alarms are clinically relevant, they can be further differentiated into clinically relevant or not relevant (e. g., inadequate thresholds).

\section{False alarm rates}

There are several studies in the medical literature about monitoring alarms in anesthesiology and intensive care medicine. Lawless suggested that $94 \%$ of all alarms in a pediatric intensive care unit (PICU) were clinically irrelevant [15]. Tsien and Fackler also found that $92 \%$ of alarms were false alarms in their observation in a PICU [16]. In both studies, all alarms were recorded by the nursing staff, who also assessed their relevance and validity. O'Carroll reported that only 8 of 1,455 alarms were caused by potentially life-threatening situations [17]. An observation by Siebig and co-workers showed that these results are not limited to the PICU. These authors digitally recorded all the alarms for 38 patients on a 12-bed medical ICU and retrospectively assessed their relevance and validity: Only $17 \%$ of the alarms were relevant, with $44 \%$ being technically false [18]. Chambrin et al. conducted a multicenter study in 1999, including 131 medical ICU patients. The medical staff recorded all alarms, which were assessed according to their relevance and the reaction of the medical staff. Twenty-six percent of the alarms had marginal consequences, for example leading to re-positioning of sensors. In only $6 \% \mathrm{did}$ the alarm lead to a call for a doctor. Seventeen percent were the result of technical problems and $24 \%$ were caused by staff manipulation [14].

In contrast to ICU observations, there are only a few studies about false alarms in perioperative settings. Comparison between the ICU and the operating room (OR) is limited in part because ICU patients are only sedated and not anesthetized, causing higher rates of patient movement artifacts. Furthermore, in the OR, changes in patients' conditions often occur much more rapidly than in the ICU because of changes in the depth of anesthesia and surgical manipulation (e. g., extensive blood loss).

Schmid et al. [19] studied perioperative alarms in a highly complex surgical setting and included 25 patients undergoing elective cardiac surgery with extracorporeal circulation. All patient monitor and anesthesia workstation alarms were digitally recorded. Additionally, the anesthesiology workplace was videotaped from two angles to allow better assessment of external influences, retrospectively. During 124 hours of monitoring, 8,975 alarms were recorded: 7,556 alarms were hemodynamic alarms, 1,419 alarms were ventilation-related. This 
corresponded to $359 \pm 158$ alarms per procedure (1.2 alarms/minute). The reaction time to the alarms was on average 4 seconds. Of all the alarms, $96 \%$ were caused by threshold violations. Of the 8,975 alarms, 6,386 were classified as serious and life-threatening and analyzed further: 4,438 (70\%) of these were labeled as valid, 1,948 (30\%) were caused by artifacts; 1,735 (39\%) of the valid alarms were classified as relevant, 2,703 (61\%) were not relevant.

These results supported earlier studies in less complex settings. Seagull and Sanderson surveyed perioperative alarms in different surgical disciplines (arthroscopic, cardiac surgery, abdominal surgery, and neurosurgery) with 6 cases in each discipline. The authors found $72 \%$ of alarms had no clinical consequences [20]. A study by Kestin et al. [21] included 50 pediatric patients (1 month to 10 years old) in the OR of a pediatric hospital (pediatric surgery, eye surgery, dental surgery, orthopedic surgery) and also found that $75 \%$ alarms had no therapeutic consequences ( 1 alarm per 4.5 minutes on average). Only $3 \%$ of all alarms indicated critical situations [21]. However, the studies by Kestin [21] and Seagull [20] were limited by the fact that 5 and 6 different monitors, respectively, were used in these observational studies.

\section{Artifacts: a common source of false alarm}

Many false alarms are caused by artifacts. The main sources of artifact are well known and are of physiological and non-physiological origin. Most of these artifacts directly influence the measured signals [22], leading to incorrect measurements and this, in turn, triggers the alarm. The most common artifacts and their sources are listed in Table 1.

\section{Consequences of false alarms}

The story of "the shepherd who cried wolf" appears in Aesop's Fables and, with some minor variations, can be found in the folklore of many different cultures. "One day, just to stir up excitement, the shepherd boy rushed down from the pasture, crying 'Wolf! Wolf!' The villagers heard the alarm and came running to help chase the marauder away, only to find the sheep peaceful and no wolf in sight. But there came a day when a wolf really came. The boy screamed and called for help. But all in vain! The neighbors, supposing him to be up to his old tricks, paid no heed to his cries, and the wolf devoured the sheep!" [23].

Based on this Fable, Breznitz formed the phrase "Crying-Wolf-Phenomenon" for the desensitization caused by high false alarm rates, with the possible consequence of ignoring relevant alarms [24]. The high incidence of false alarms in anesthesiology and intensive care medicine is not only a disturbance but a risk factor when relevant alarms in critical situations are ignored.
That this phenomenon is not limited to monitoring alarms was impressively demonstrated by the attack of the Japanese air force on the United States Navy at Pearl Harbor, Hawaii on December 7, 1941. Despite a valid advance warning by the new radar technology, no appropriate reaction followed. The simultaneous report of a contact and the destruction of an enemy submarine also did not lead to a reaction because the commanding admiral wanted to wait for confirmation due to frequent false alarms [25].

Various studies have shown that anesthesiologists' reaction times to alarms increases in situations where there is low alarm validity [26,27]. The annoyance from false alarms may also lead to complete inactivation of alarms or to inappropriately wide threshold settings by the clinical user to limit alarms as much as possible. Thereby, the 'mesh of the alarm-net' gets wider and the risk of missed relevant alarm increases [28].

\section{Medical staff and alarms}

Alarms in the ICU and in the OR frequently lead to sound levels up to $70 \mathrm{~dB}(\mathrm{~A})$. This level corresponds to heavy traffic. Sound levels up to $90 \mathrm{~dB}$ are not rare [29]. In a study by Hagerman et al., 94 patients with chest pain were retrospectively distributed into a good and poor acoustic group. Acoustics were altered during the study period by changing the ceiling tiles throughout the ICU from sound-reflecting (poor acoustics) to soundabsorbing tiles (good acoustics) of similar appearance. The patients were asked to complete a questionnaire about the quality of care. The patients considered the staff attitude was much better in the good acoustics period [30]. Increased sound levels caused by alarms can impact on the health of the medical personnel. In 1988, Topf and Dillon demonstrated the relationship between increased sound levels in ICUs and burn-out syndromes in ICU nurses [31].

\section{Patients and alarms}

For undisturbed night-sleep, sound levels below $40 \mathrm{~dB}(\mathrm{~A})$ are recommended. As sound levels on the ICU are frequently above this level, sleep deprivation in ICU patients is well recognized [32]. Sleep deprivation in ICU patients leads to an impairment of the immune response and increased sympathetic nervous system activity: Catecholamine secretion increases heart rate, metabolism, and oxygen consumption [33]. Frequent arousal from sleep may lead to cardiac arrhythmias in patients with pre-existing heart disease but also in healthy patients [34]. Minckley reported significantly increased opioid needs when noise levels were high in her observation of 644 postoperative patients [35]. In the study by Hagerman et al, patients during the good acoustic (sound-absorbing ceiling tiles) period had lower pulse 


\begin{tabular}{|c|c|c|}
\hline Signal & Artifact source & Parameter \\
\hline \multicolumn{3}{|l|}{ Ventilatory alarms } \\
\hline Pulse oximetry & $\begin{array}{l}\text { Movement } \\
\text { Injection of contrast dye } \\
\text { Interruption of blood-flow by non-invasive blood pressure } \\
\text { measurement } \\
\text { Ambient light }\end{array}$ & $\begin{array}{l}\text { Oxygen saturation } \\
\text { Pulse frequency }\end{array}$ \\
\hline Capnography & $\begin{array}{l}\text { Occlusion of } \mathrm{CO}_{2} \text {-line (by kinking or built up fluid) } \\
\text { Ventilator circuit leakage } \\
\text { Atmospheric pressure variations } \\
\text { Suctioning } \\
\text { Dead space in measurement circuit }\end{array}$ & $\begin{array}{l}\text { End-tidal } \mathrm{CO}_{2} \\
\text { Inspired } \mathrm{CO}_{2} \\
\text { Respiratory rate }\end{array}$ \\
\hline \multicolumn{3}{|l|}{ Hemodynamic Alarms } \\
\hline EKG & $\begin{array}{l}\text { Electrosurgical interference } \\
\text { Power-line interference } \\
\text { Movement artifacts (patient movement, positioning) } \\
\text { Electrode instability or electrode distortion } \\
\text { EMG/neuromonitoring interference } \\
\text { Incorrect connection or lead contact } \\
\text { Pacing/defibrillation } \\
\text { Abnormally tall T-waves mistaken as QRS-complex } \\
\text { MRI interference }\end{array}$ & $\begin{array}{l}\text { Heart rate, } \\
\text { ST-values } \\
\text { Arrhythmia detection }\end{array}$ \\
\hline Non-invasive blood pressure & $\begin{array}{l}\text { Movement } \\
\text { Inadequate size or cuff position } \\
\text { Compression of cuff by external forces (surgeon or equipment } \\
\text { pressing against the cuff } \\
\text { Kinked cuff tubing and leaking cuff bladder }\end{array}$ & $\begin{array}{l}\text { Systolic, diastolic blood pressure } \\
\text { Mean arterial pressure }\end{array}$ \\
\hline \multicolumn{3}{|l|}{ Other Alarms } \\
\hline Temperature & Dislocated sensor & Temperature \\
\hline
\end{tabular}

EKG: electrocardiogram, EMG: electromyogram, MRI: magnetic resonance imaging, $\mathrm{CO}_{2}$ : carbon dioxide

amplitude values than those in the bad acoustic group; patients in the bad acoustics group also had higher rates of re-hospitalization after 1 (18\% vs. $10 \%)$ and 3 months (48 \% vs. $21 \%$ ) [30]. In a recent study, Van Rompaey et al. found reduced rates and later onset of delirium in patients who slept with earplugs at night [36].

\section{Technical approaches for false alarm reduction}

Essentially, there are three technical approaches to help reduce false alarms: (1) Improving signal extraction (prevention or detection of artifacts); (2) improving algorithms for alarm generation; (3) improving alarm validation. An algorithm for alarm generation can be based on a single parameter (e. g., heart-rate or mean arterial pressure) or on several parameters simultaneously (e. g., heart rate detection from elecrocardiogram [EKG], pulse oximetry oxygen saturation $\left[\mathrm{SpO}_{2}\right]$ and arterial line). Most devices are equipped with alarms based on a single parameter. In recent years, different approaches for false alarm reduction have been developed.

\section{Phase specific settings}

Observations, especially in surgical settings, have shown that different phases of the surgical procedure are characterized by different numbers and types of false alarms and also different patterns of alarms and specific reactions by the medical staff (e. g., during induction and emergence of anesthesia, during extracorporeal circulation, or single lung ventilation, or suctioning of patients). Schmid et al. found different characteristic patterns and density of alarms in 4 different intraoperative phases (beginning of surgery, start of extracorporeal circulation [ECC], end of ECC, end of surgery) [19].

Seagull and Sanderson also differentiated three different phases in anesthesia procedures (introduction, maintenance and emergence) and found characteristic patterns of alarms and alarm reactions in each phase [20].

This knowledge could be used for the development of phase specific settings to reduce false alarms (e. g., for specific settings for surgery or on the ICU).

\section{Integrated validation of alarms (cross checking)}

Matching of different parameters can be used for the reduction of false alarms: e. g., a 'ventricular fibrillation' alarm can be assumed to be false in the presence of undisturbed pulse oximetry and arterial blood pressure waveforms. Aboukhalil et al. [37] were able to reduce the incidence of false arrhythmia-related alarms from $42.7 \%$ 
to $17.2 \%$ in an offline application on a database of 447 patients (5,386 arrhythmia alarms). However, $9.7 \%$ of the true cases of ventricular fibrillation were not detected; this situation is inadmissible for a lethal arrhythmia. Removal of ventricular arrhythmias from the algorithm still resulted in reduction in the incidence of false alarms to $22.7 \%$ [37].

\section{Implementation of time delays}

Görges et al. [38] showed in an ICU setting and Schmid et al. [19] in an intraoperative setting that a great number of false alarms are caused by only mild threshold violations of short duration. In an offline validation, Görges et al. showed that the implementation of a 14-second delay reduced false alarms by $50 \%$; a 19 -second delay reduced false alarms by $67 \%$. However, a simple delay carries the risk of unrecognized critical situations of short duration (e.g., short self-limiting tachycardia). The implementation of a graduated delay brings additional safety and flexibility to that approach. First, severe deviations are alarmed faster; this results in improved patient safety. Second, the graduation offers the possibility of a prolonged delay (more than 14 seconds) in cases of only moderate and clinically not-relevant deviations. However, studies using such an approach are still missing.

\section{Statistical approaches for false alarm reduction}

Improved signal extraction is an essential approach for reduction of false alarms caused by artifacts. Several approaches have been developed over the last decades.

\section{Autoregressive models and self-adjusting thresholds}

An autoregressive model describes measurement values as a linear transformation and integrates previous values plus a random error. An autoregressive model is appropriate for the observation of values in steady-state and for alarm generation caused by deviation from steady-state. It is also used for generation of self-adjusting thresholds, because of integration of the individual patient's condition. However, self-adjusting thresholds always have to be elaborated and confirmed by the user [9].

\section{Statistical process control}

Statistical approaches are commonly used in alarm systems to detect 'out-of-control' states in a process. The original applications were in industrial production processes but they are also used for alarm generation. Kennedy used a process control approach to detect the onset of changes in systolic blood pressure [39]. The algorithm was tested on an existing database and detected $94 \%$ of changes correctly, whereas anesthesiologists only detected $85 \%$.

\section{Median filters}

The median filter is a non-linear, signal processing method used for removal of short-term noise in measurement signals without influencing the baseline signal. For this purpose, the median is calculated for a defined interval. Thus, the signal is 'smoothed' and short noises, such as movement artifacts or interference from electrosurgery, are eliminated. This method is limited in longlasting interferences that exceed the adjusted duration of the filter. Mäkivirta et al. [40] evaluated the effectiveness of a combination of a "short" (15 seconds) and a "long" (2.5 minutes) filter in a database of 10 cardiac surgery patients. The use of the filter increased the alarms that had therapeutic consequences from 12 to $49 \%$; the authors declared that no relevant alarms were missed.

\section{Artificial intelligence}

Although statistical approaches are predominantly used for the reduction of artifacts, artificial intelligence offers the possibility of integrating more complex contexts. This approach tries to validate alarms by imitating human thinking. Artificial intelligence can be embedded into decision-making systems. A paper by Imhoff and Kuhls provides an overview of artificial intelligence use in intensive care monitoring [9].

\section{Rule-based expert systems}

Rule-based expert systems are based on an integrated expert knowledge database. Some early rule-based expert systems were developed for medical use in the 1970s (MYCIN-System, ONCOCIN-System) [41]. These early systems applied expert knowledge from a database into a new context and simulated expert decisions in oncology and therapy of infectious diseases. In 1993, Sukuvaara et al. [42] developed an alarm system for the detection of hypovolemia, hyperdynamic circulation, left-heart failure, and hypoventilation. Although results showed that rulebased expert systems work well in the context of pathologic conditions, they have not been introduced into the clinical arena. The expansion of rule-based expert systems by so-called machine-learning is possible, whereby the pre-existing database is updated by actual patient data.

\section{Neural networks}

Neural networks were developed to imitate the neuronal process of human thinking. They are able to anticipate the presence of diseases on the basis of advance information (e. g., hemodynamic data from a myocardial infarction study group). Baxt and Skora [43] developed a neural network for early detection of myocardial infarction in patients admitted to a hospital with chest pain. The system was "trained" to detect specific changes in patients with myocardial infarction by implementation of a database (350 patients, 120 of whom had myocardial 
infarction). The results showed that the neural network was able to detect or to exclude infarction with a sensitivity and specificity of $96 \%$. The doctors at the respective emergency department achieved an average sensitivity of $73.3 \%$ and specificity of $81.1 \%$. Neural networks have also been used for alarm generation in anesthesia ventilators [44].

\section{Fuzzy logic}

Fuzzy logic was introduced by Zadeh in the 1960s [45]. A common problem in clinical routine is the aim for objectivity and precision when the information does not allow an explicit conclusion. Fuzzy logic allows diffuse processing of exact data. Fuzzy logic is widely used in industry (e. g., for picture stabilization in cameras). Goldman and Cordova [46] demonstrated a patient monitor that was able to diagnose a simulated cardiac arrest by evaluation of EKG, capnography, and arterial blood pressure using fuzzy logic.

\section{Bayesian networks}

Bayesian networks have been used for estimation of event occurrence. In patient monitoring, they can be used for decision support. Laursen developed software for cardiac event detection [47]. The software continuously compared different physiologic parameters and their changes; thus, it was possible to check values against each other for plausibility and to anticipate cardiac events.

\section{Conclusion}

Medical progress has led to obvious improvements in ICU and perioperative monitoring over recent decades. With the increase in 'monitorable' parameters, rates of alarms have also increased. But technical progress has rarely affected the rates of false alarms. In addition to noise-related increase in burn-out rates, false alarms lead to desensitization of medical staff to alarms with the risk of critical situations potentially being ignored despite correct alarming. Patients are also directly affected by alarm-related sleep disorders with subsequent development of delirium and increased sympathetic nervous system activity and catecholamine secretion. In recent years, many promising approaches using statistical methods and artificial intelligence have been developed for the reduction of false alarms without obvious changes in false alarm rates in our clinical reality.

\section{Competing interests}

The authors declare that they have no competing interests.

List of abbreviations used

ECC: extracorporeal circulation; EKG: electrocardiogram; EMG: electromyogram; ICU: intensive care unit; MRI: magnetic resonance imaging; $\mathrm{PICU}$ : pediatric intensice care unit; $\mathrm{SpO}_{2}$ : pulse oximetry oxygen saturation.

Published: 19 March 2013
References

1. Tsunami Alarm System. [http://www.tsunami-alarm-system.com/en/index. html] Accessed Oct 2012

2. Billings CE, Raynard WD: Human factors in aircraft incidents: results of a 7-years study. Aviat Space Environ Med 1984, 55:960-965.

3. Kowalski-Trakofler KM, Vaught C, Mallett LG: Safety and health training for an evolving workforce: an overview from the mining industry. Information Circular 2004, 9474:1-15.

4. International Organization for Standardization: IEC-ISO 60601-1-11 2010. [http://www.iso.org/iso/home/store/catalogue_tc/catalogue_detail. htm?csnumber=45605]. Accessed August 2012

5. International Organization for Standardization: IEC 80001-1:2010 2010 [http://www.iso.org/iso/home/store/catalogue_tc/catalogue_detail. htm?csnumber=44863]. Accessed August 2012.

6. Tinker JH, Dull DL, Caplan RA, Ward RJ, Cheney FW: Role of monitoring devices in prevention of anesthetic mishaps: A closed claims analysis. Anesthesiology 1989, 71:541-546.

7. Cooper JB, Newbower RS, Kitz RJ: An analysis of major errors and equipment failures in anesthesia management: considerations for prevention and detection. Anesthesiology 1984, 60:34-42.

8. Flight Safety Foundation: Flight Safety Digest - The Human Factors Implications for Flight Safety of Recent Developments in the Airline Industry 2003, [http://flightsafety.org/fsd/fsd_mar-apr03.pdf] Accessed Oct 2012

9. Imhoff M, Kuhls S: Alarm algorithms in critical care monitoring. Anesth Analg 2006, 102:1525-1537.

10. Loeb RG: A measure of intraoperative attention to monitor displays. Anesth Analg 1993, 76:337-341.

11. Morris RW, Montano SR: Response times to visual and auditory alarms during anaesthesia. Anaesth Intensive Care 1996, 24:682-684.

12. Imhoff M, Kuhl S, Gather U, Fried R: Smart alarms from medical devices in the OR and ICU. Best Pract Res Clin Anaesthesiol 2009, 23:39-50.

13. Block FE, Rouse JD, Hakala M, Thomson CL: A proposed new set of alarm sounds which satisfy standards and rationale to encode source information. J Clin Monit 2000, 16:541-546.

14. Chambrin MC, Raveaux P, Calvelo-Aros D, Jaborska A, Chopin C, Boniface B: Multicentric study of monitoring alarms in the adult intensive care unit (ICU): A descriptive analysis. Intensive Care Med 1999, 25:1360-1366.

15. Lawless ST: Crying Wolf: False alarms in a pediatric intensive care unit. Crit Care Med 1994, 22:981-985.

16. Tsien $\mathrm{CL}$, Fackler JC: Poor prognosis for existing monitors in the intensive care unit. Crit Care Med 1997, 25:614-619.

17. O'Carroll TM: Survey of alarms in an intensive care unit. Anaesthesia 1986, 41:742-744.

18. Siebig S, Kuhls S, Imhoff M, Langgartner J, Reng M, Schölmerich J, Gather U, Wrede CE: Collection of annotated data in a clinical validation study for alarm algorithms in intensive care - A methodologic framework. J Crit Care 2010, 25:128-135.

19. Schmid F, Goepfert MS, Kuhnt D, Eichorn V, Diedrichs S, Reichenspurner H, Goetz AE, Reuter DA: The wolf is crying in the operating room: patient monitor and anesthesia workstation alarming patterns during cardiac surgery. Anesth Analg 2011, 112:78-83.

20. Seagull FJ, Sanderson PM: Anesthesia alarms in context: An observational Study. Hum Factors 2001, 43:66-78.

21. Kestin IG, Miller BR, Lockhart CH: Auditory alarms during anesthesia monitoring. Anesthesiology 1988, 69:106-109.

22. Takla G, Petre JH, Doyle JD, Horibe M, Gopakumaran B: The problem of artefacts in patient monitor data during surgery: A clinical and methodological review. Anesth Analg 2006, 103:1196-1204.

23. Aesop: The Shepherd Boy and the Wolf. In: Aesops Fables. Watermill Classic Edition. NJ Watermill Press, Mahwah; 1985:116-117.

24. Breznitz S: Cry wolf: The Psychology of False Alarms. Psychology Press, New York; 1984

25. Barkeley AW, Cooper J, George WF: Congressional investigation of the pearl harbor attack. US Government, Washington; 1946:141-143.

26. Westenskow DR, Orr J, Simon FH, Bender HJ, Frankenberger H: Intelligent alarms reduce anaesthesiologist's response time to critical faults. Anesthesiology 1992, 77:1074-1079.

27. Bliss JP, Dunn MC: Behavioural implications of alarm mistrust as a function of task workload. Ergonomics 2000, 43:1283-1300.

28. Block FE, Nuutinen L, Ballast B: Optimization of alarms: A study on alarm 
limits, alarm sounds and false alarms intended to reduce annoyance. $J$ Clin Monit 1999, 15:75-83.

29. Kam PCA, Kam AC, Thompson JF: Noise pollution in the anaesthetic and intensive care environment. Anaesthesia 1994, 49:982-986.

30. Hagerman I, Rasmanis G, Blomkvist V, Ulrich R, Eriksen CA, Theorell T: Influence of intensive coronary care acoustics on the quality of care and physiological state of patients. Int J Cardiol 2005, 98:267-270.

31. Topf $M$, Dillon E: Noise induced stress as a predictor of burnout in critical care nurses. Heart Lung 1988, 17:567-573.

32. Aaron JN, Carlisle CC, Carskadon MA, Meyer TJ, Hill NS, Millman RP: Environmental noise as a cause of sleep disruption in an intermediate respiratory care unit. Sleep 1996, 19:707-710.

33. Hansell HN: The behavioural effects of the noise on men: The patient with "intensive care psychosis". Heart Lung 1984, 13:59-65.

34. Smith R, Johnson L, Rothfeld D, Zir L, Tharp B: Sleep and cardiac arrythmias. Arch Intern Med 1972, 130:751-753.

35. Minckley BB: A study of noise and its relationship to patient discomfort in the recovery room. Nurs Res 1968, 17:247-250.

36. Van Rompaey B, Elseviers MM, Van Drom W, Fromont V, Jorens PG: The effect of earplugs during the night on the onset of delirium and sleep perception: a randomized controlled trial in intensive care patients. Crit Care 2012, 16:R73

37. Aboukhalil A, Nielsen L, Saeed M, Mark RG, Clifford GD: Reducing false alarm rates for critical arrhythmias using the arterial blood pressure waveform. J Biomed Inf 2008, 41:442-451.

38. Görges M, Markewitz BA, Westenskow DR: Improving alarm performance in the medical intensive care unit using delays and clinical context. Anesth Analg 2009, 108:1546-1552.
39. Kennedy RR: A modified trigg's tracking variable as an "advisory" alarm during anesthesia. Int J Clin Monit Comput 1995, 12:197-204.

40. Mäkivirta A, Koski E, Kari A, Sukuvaara T: The median filter as a processor for a patient monitor limit alarm system in intensive care. Comput Methods Programs Biomed 1991, 34:139-144.

41. Shortliffe EH, Davis R, Axline SG, Buchanan BG, Green CC, Cohen SN: Computer-based consultations in clinical therapeutics: Explanation and rule acquisition capabilities of the MYCIN-System. Comput Biomed Res 1975, 8:303-320.

42. Sukuvaara T, Koki EM, Mäkivirta A, Kari A: A knowledge-based system for monitoring cardiac operated patients - technical construction and evaluation. Int J Clin Monit Comput 1993, 10:117-126.

43. Baxt WG, Skora J: Prospective validation of artificial neural network trained to identify acute myocardial infarction. Lancet 1996, 347:12-15.

44. Orr JA, Westenskow DR: A breathing circuit alarm system based on neural networks. J Clin Monit 1994, 10:101-109.

45. Zadeh LA: Fuzzy sets. Inform Control 1965, 8:338-353.

46. Goldman JM, Cordova MJ: Advanced clinical monitoring: Considerations for real-time hemodynamic diagnostics. Proc Annu Symp Comput Appl Med Care 1994:752-756

47. Laursen P: Event detection on patient monitoring data using causal probabilistic networks. Method Inform Med 1994, 33:111-115.

doi:10.1186/cc12525

Cite this article as: Schmid F, et al.: Patient monitoring alarms in the ICU and in the operating room. Critical Care 2013, 17:216. 